\title{
DYNAMICAL SYSTEMS SATISFYING CERTAIN STABILITY AND RECURSIVE CRITERIA
}

\author{
RONALD A. KNIGHT
}

\begin{abstract}
A necessary and sufficient condition for the various characteristic zero concepts to coincide is given. We prove that on locally compact spaces a flow of characteristic 0 and the center of a flow of characteristic $0^{+}$are almost of characteristic $0 \pm$. Poisson stable flows and nonwandering flows of the zero characteristics are characterized in terms of the prolongation, prolongational limit, orbit closure, limit, and weak attraction relations. Examples showing the results are sharp are given.
\end{abstract}

1. Introduction. Central to the qualitative theory of motions for abstract dynamical systems are the classical notions of central, Poisson stable, recurrent, and nonwandering motions and attractive, orbitally stable, and minimal sets. Considerable effort has been directed toward qualitatively classifying such motions. Ahmad and Knight introduced the zero characteristic classes of flows each satisfying several of these classical concepts in [ 2 and 5 ], respectively. In this paper we classify and characterize these flows using the classical notions and the prolongation, prolongational limit, orbit closure, limit, orbit, and weak attraction relations denoted, respectively, by $D, J, K, L, C$, and $A_{w}$.

We recapitulate a few definitions for the convenience of the reader. Throughout the remainder of this paper $(X, \pi)$ denotes a given flow on a Hausdorff space $X$. A point $x$ of $X$, motion $\pi_{x}$, and trajectory $C(x)$ are called positively recurrent if and only if, given any neighborhood $U$ of $x$, there is a $T \geq 0$ such that $U \cap y[0, T] \neq \emptyset$ for every $y \in C(x)$. A point $x$ of $X$ is said to be positively Poisson stable (nonwandering) provided $x \in L^{+}(x)\left(x \in J^{+}(x)\right.$, or equivalently, $\left.x \in J^{-}(x)\right)$. A point $x$ of $X$ is positively divergent (dispersive) whenever $L^{+}(x)=\emptyset\left(J^{+}(x)=\emptyset\right)$. A set $M \subset X$ is said to be positively stable if each neighborhood $U$ of $M$ contains a neighborhood $V$ such that $C^{+}(V) \subset U$. The negative and bilateral versions of the notions above are defined similarly and the term "positively" is frequently omitted. A set $M \subset$ $X$ is called positively (negatively) minimal or minimal if and only if it is a closed positively (negatively) invariant set or invariant set, respectively, and contains no nonempty proper subset with these respective properties. The center of $(X, \pi)$ is the maximal nonwandering subflow of $(X, \pi)$.

The following theorems are not widely known and are used sufficiently often in this paper for us to state them (see [3, 7, and 10]). In these theorems and throughout the remainder of this paper we shall say that an invariant set or subflow of $(X, \pi)$ has a property $P$ whenever each of its points or trajectories enjoys property $P$.

Received by the editors May 26, 1981 and, in revised form, October 1, 1981.

1980 Mathematics Subject Classification. Primary 34C35; Secondary 54H20.

Key words and phrases. Center, divergent, dispersive, dynamical system, flow, minimal, nonwandering, Poisson stable, recurrent, stability, weak attraction, zero characteristic.

(c) 1982 American Mathematical Society 0002-9939/81/0000-0459/\$02.50 
THEOREM 1.1 (AHMAD-SARABIA). A flow is nonwandering if and only if $D^{+}$ $=D^{-}$.

The space $X$ in the following two theorems is locally compact.

THEOREM 1.2. A flow is recurrent if and only if it is positively (negatively) Poisson stable.

THEOREM 1.3. If $(X, \pi)$ is nonwandering, then the set of bilaterally Poisson stable points is dense in $X$.

2. Flows of characteristics $0^{+}, 0^{-}, 0^{ \pm}$, and 0 . Recall that a flow is of characteristic $0^{+}\left(0^{-}, 0,0^{ \pm}\right)$provided $D^{+}=K^{+}\left(D^{-}=K^{-}, D=K, D^{+}=\right.$ $K^{+}$and $\left.D^{-}=K^{-}\right)$. In [1 and 6] Ahmad and Knight proved that the zero characteristic concepts are equivalent whenever the phase space is compact. A key property of such flows is that no point diverges in either direction. Examples showing that these concepts are distinct are easily constructed when divergent points exist (see Example 1 and [5]). Our first theorem generalizes these results to arbitrary spaces.

THEOREM 2.1. If no point is positively or negatively divergent, then the characteristic $0^{+}, 0^{-}, 0^{ \pm}$, and 0 properties are pairwise equivalent.

ProOF. First, let $(X, \pi)$ be of characteristic $0^{+}$. Since $L^{-}(x) \neq \emptyset$ for every $x \in X$, we have $L^{-}(x)=L^{+}(x)=K(x)$ by Lemma 4.5 of [1]. Consequently, each point $x$ of $X$ is nonwandering and we have $D^{+}(x)=D^{-}(x)$ by the Ahmad-Sarabia Theorem 1.1 which yields $J^{-}(x)=D^{-}(x)=D^{+}(x)=J^{+}(x)=L^{+}(x)=$ $L^{-}(x)$. The flow is thus also of characteristics $0^{-}, 0^{ \pm}$, and 0 . The dual argument implies that $(X, \pi)$ is of the other three characteristics whenever it is of characteristic $0^{-}$or $0^{ \pm}$. Finally, let $(X, \pi)$ be of characteristic 0 . Then, since $L^{-}(x) \neq$ $\emptyset \neq L^{+}(x)$ for each $x$ in $X$, Corollary 3.1 of [6] applies yielding the desired results. The proof is complete.

THEOREM 2.2. Let $(X, \pi)$ be of characteristic $0^{+}, 0^{-}, 0^{ \pm}$, or 0 . A necessary and sufficient condition for $(X, \pi)$ to be of characteristics $0^{+}, 0^{-}, 0^{ \pm}$, and 0 is that each point be either bilaterally dispersive or else bilaterally nondivergent.

Proof. First, let $(X, \pi)$ be of characteristics $0^{+}, 0^{-}, 0^{ \pm}$, and 0 . Then, the desired result follows from Corollary 3.1 of [6]. Conversely, let $(X, \pi)$ be of characteristic $0^{+}$. If some point $x$ of $X$ is bilaterally dispersive, then $J^{+}(x)=$ $J^{-}(x)=L^{+}(x)=L^{-}(x)=\emptyset$. On the other hand, let some point $x$ of $X$ be bilaterally nondivergent. Then, $J^{+}(x)=L^{+}(x)=L^{-}(x) \subset J^{-}(x)$ by Lemma 4.5 of [1]. Also, for any point $y$ in $J^{-}(x)$ we have $x \in J^{+}(y)=L^{+}(y)=L^{-}(y) \subset$ $J^{-}(y)$, and hence, $y \in J^{+}(x)$. Consequently, $J^{+}(x)=J^{-}(x)$ and $(X, \pi)$ is of characteristics $0^{-}, 0^{ \pm}$, and 0 . The dual argument yields the dual assertion. A flow of characteristic $0^{ \pm}$is always of the other zero characteristics. Whenever $(X, \pi)$ is of characteristic 0 , Corollary 3.1 of [6] implies the desired properties. The proof is complete.

COROLLARY 2.2.1. If $(X, \pi)$ satisfies each of the zero characteristic conditions, then all nonwandering motions are nondivergent central motions and all wandering motions are dispersive. 
Next, we give an example of a central flow of characteristic 0 with no recurrent points and phase space locally compact. It does have dense subflows of each zero characteristic even though it satisfies none of these properties except characteristic 0 .

EXAMPLE 1. A toral flow $(T, \pi)$ is given in Example 4.06 of [11] consisting of one critical point $p$ and dense regular trajectories. All but two regular trajectories are bilaterally dense in $T$. One of the remaining regular trajectories $C(x)$ satisfies $L^{+}(x)=T$ and $L^{-}(x)=\{p\}$ whereas the other $C(y)$ satisfies $L^{+}(y)=\{p\}$ and $L^{-}(y)=T$. The subflow on the punctured torus $X=T \backslash\{p\}$ is of characteristic 0 but not of characteristics $0^{+}, 0^{-}$, or $0^{ \pm}$. However, the flow has such subflows with phase spaces dense in $X$. We shall see in Theorem 2.3 that this is always the case on locally compact spaces. There are subflows on nonlocally compact subsets of $X$ which do not enjoy this property. No point of $X$ is recurrent and each point of $X$ is central. Theorem 2.5 characterizes such recurrent flows.

We now show that every flow of characteristic 0 on a locally compact phase space is almost of characteristic $0^{ \pm}$.

THEOREM 2.3. Let $X$ be locally compact and $(X, \pi)$ be of characteristic 0 . Then, $(X, \pi)$ has a subflow of characteristic $0^{ \pm}$on a dense subset of $X$.

ProOF. Let $Y$ be the set of nonwandering points of $X$. The set $Y$ is closed in $X$ so that the limit set $L_{Y}(x)$ of a point $x$ in $Y$ is $L(x) \cap Y$. For any $x \in X$, $J(x)=K(x)=K_{Y}(x)=J_{Y}(x)$. The set of bilaterally Poisson stable points are dense in $Y$ by the Central Motion Theorem 1.3. The only points of $X$ not contained in $Y$ are the bilaterally dispersive points on which the flow is of characteristic $0^{ \pm}$. Hence, $(X, \pi)$ contains a dense subflow of characteristic $0 \pm$, namely, the subflow on $\left\{x: J^{+}(x)=L^{+}(x)=L^{-}(x)\right\}$ (see [6, Theorem 6]). The proof is complete.

No result like this theorem holds for flows of characteristic $0^{+}$or $0^{-}$, however, such a result does hold on the center of a flow on a locally compact phase space.

THEOREM 2.4. Let $X$ be locally compact and $(X, \pi)$ be of characteristic $0^{+}$ $\left(0^{-}\right)$. Then, the center $M$ of $(X, \pi)$ contains a subflow of characteristic $0^{ \pm}$on $a$ dense subset of $M$.

Proof. The center $M$ is a closed, and hence, locally compact subset of $X$. The set of bilaterally Poisson stable points are dense in $M$ by the Central Motion Theorem 1.3. For each bilaterally Poisson stable point $x$ of $M$ we have $J^{+}(x)=$ $L^{+}(x)=L^{-}(x) \subset J^{-}(x)$. Suppose that $y \in J^{-}(x)$. Then, $x \in J^{+}(y)=L^{+}(y)$. By Lemma 4.5 of $[1]$ the set $L^{+}(y)$ is positively minimal and $L^{+}(x)=L^{+}(y)$. Since $y \in M$ we have $y \in L^{+}(y)=L^{-}(x)$. Hence, $J^{-}(x)=L^{+}(x)$ and the set of bilaterally Poisson stable points form a dense subflow of characteristic $0 \pm$. The proof is complete.

Evidently, the succeeding theorem follows from several preceeding theorems and Ura's stability theorem (see $[4,1.11$, II]).

THEOREM 2.5. Let $X$ be locally compact and $(X, \pi)$ be of characteristic $0^{+}, 0^{-}$, or 0 . The following conditions are pairwise equivalent:

(a) The center is of characteristic $0 \pm$.

(b) The center is recurrent.

(c) Each semilimit set of the center is bilaterally stable. 
Flows on locally compact spaces with all orbits either periodic or critical are of characteristic 0 if and only if the critical points are bilaterally stable (see [9]). The following theorem is an easy consequence.

THEOREM 2.6. Let $X$ be locally compact and let each orbit be periodic or critical. Then, $(X, \pi)$ contains a subflow of characteristic $0^{ \pm}$on an open dense subset of $X$.

COROLLARY 2.6.1. Let $X$ be locally compact. If the center $M$ consists of compact orbits, then it contains a subflow of characteristic $0^{ \pm}$on a dense subset of $M$.

3. Poisson stability and characteristic $0^{+}, 0^{-}, 0^{ \pm}$, and 0 . In this section we classify and characterize classes of Poisson stable and nonwandering flows of zero characteristic in terms of dynamic relations. We examine first positively Poisson stable flows and then bilaterally Poisson stable flows. We include known and easily proven results for completeness.

THEOREM 3.1. The following conditions are pairwise equivalent:

(a) $(X, \pi)$ is positively Poisson stable.

(b) $K^{+}=K$.

(c) $K^{+}=L$.

(d) $K^{+}=L^{+}$.

(e) $K=L^{+}$.

Proof. If condition (a) holds, then $C \subset L^{+}$implies $K \subset L^{+} \subset K^{+}$or $K^{+}=K$. If condition (b) holds, then $K^{+}$is invariant implying that $C \subset L^{+} \subset$ $L$, and hence, $K^{+}=K=L$. If condition (c) holds, then again we have $K^{+}$ invariant so that $C \subset L^{+}$. Thus, $L^{-} \subset L^{+}$and $L=K^{+} \subset L^{+}$, and so, $K^{+}=L^{+}$. If condition (d) holds, then as before the invariance of $K^{+}$implies that $C \subset L^{+}$and $K \subset L^{+}=K^{+}$so that $K=L^{+}$. Finally, if condition (e) holds, $C \subset K \subset L^{+}$and condition (a) follows. The proof is complete.

The following theorem characterizes those flows having positively minimal orbit closures.

THEOREM 3.2. The following conditions are pairwise equivalent:

(a) $(X, \pi)$ is positively Poisson stable with positively minimal positive trajectory closures.

(b) $K^{+}=A_{w}^{+}$.

(c) $K=A_{w}^{+}$.

Proof. Each of the conditions (b) and (c) implies that $(X, \pi)$ is positively Poisson stable since $C^{+} \subset A_{w}^{+}$in each case. The equivalence of conditions (b) and (c) follows from Theorem 3.1.

We shall now show that conditions (a) and (b) are equivalent. First, let $K^{+}=$ $A_{w}^{+}$. Let $y \in L^{+}(x) \subset A_{w}^{+}(x)$ for some point $x$ of $X$. Then, $x \in L^{+}(y)$ and we have $L^{+}(x)=L^{+}(y)$. Consequently, $L^{+}(x)$ is positively minimal (see $[4,4.17, \mathrm{I}]$ ). Conversely, $K^{+}=L^{+}$, and so, let $y \in L^{+}(x)$ for some point $x$ of $X$. Then $x \in$ $L^{+}(x)=L^{+}(y)$ which means that $y \in A_{w}^{+}(x)$. On the other hand, if $y \in A_{w}^{+}(x)$, then we have $x \in L^{+}(y)$, and hence, $y \in L^{+}(x)$. Therefore, $K^{+}=A_{w}^{+}$and the proof is complete.

Our final theorem on arbitrary positively Poisson stable flows characterizes such flows of characteristic $0^{+}$. 
THEOREM 3.3. The following conditions are pairwise equivalent.

(a) $(X, \pi)$ is a nonwandering flow of characteristic $0^{+}$.

(b) $D^{+}=L^{+}$.

(c) $J^{+}=K^{+}$.

(d) $D^{-}=L^{+}$.

(e) $D^{-}=K^{+}$.

(f) $J^{-}=K^{+}$.

(g) $D^{+}=A_{w}^{+}$.

(h) $D^{-}=A_{w}^{+}$.

ProOF. Each condition implies that $(X, \pi)$ is nonwandering because each one yields either $x \in J^{+}(x), x \in J^{-}(x)$, or $x \in L^{+}(x)$ for each point $x$ of $X$. According to Theorem 1.1 we may assume that $D^{+}=D^{-}=J^{+}=J^{-}$whenever any of the conditions (a) through (h) holds. The property $J^{+}=L^{+}$is equivalent to the characteristic $0^{+}$notion (see $[4,5.2, \mathrm{I}]$ ). Therefore, conditions (a) through (f), each of which is evidently equivalent to $J^{+}=L^{+}$for nonwandering flows, are pairwise equivalent. Moreover, the last two conditions are equivalent. Consequently, in order to complete the proof of the theorem it is sufficient to prove that conditions (a) and (g) are equivalent.

Let condition (g) hold. If $y \in J^{+}(x)=D^{+}(x)=A_{w}^{+}(x)$ for some point $x$ of $X$, then $x \in L^{+}(y) \subset D^{+}(y)=A_{w}^{+}(y)$, and hence, $y \in L^{+}(x)$. Whence, $J^{+}(x)=$ $L^{+}(x)$ and condition (a) holds. Conversely, we have $L^{+}=J^{+}=D^{+}$. Let $y \in$ $D^{+}(x)$ for some point $x$ of $X$. Then, $y \in L^{+}(x)$ implies that $J^{+}(x) \subset J^{+}(y)$, and hence, that $x \in J^{+}(y)=L^{+}(y)$ and $y \in A_{w}^{+}(x)$. Thus, $D^{+}(x) \subset A_{w}^{+}(x)$. Next, let $y \in A_{w}^{+}(x)$. Then, $x \in L^{+}(y) \subset D^{+}(y)=A_{w}^{+}(y)$ so that $y \in L^{+}(x) \subset D^{+}(x)$. We have $D^{+}=A_{w}^{+}$and condition (g) holds. The proof is complete.

COROLLARY 3.3.1. Any closed invariant set with compact boundary in a nonwandering flow of characteristic $0^{+}$on a locally compact space is bilaterally stable. Moreover, the only such sets which are asymptotically stable are components of the phase space and the smallest stable set containing any point is its positive limit set.

COROLLARY 3.3.2. For any nonwandering flow of characteristic $0^{+}$we have $D=D^{+}=D^{-}=J=J^{+}=J^{-}=K^{+}=L^{+}=A_{w}^{+}$. Thus, such a flow is of characteristic $0^{ \pm}$if and only if $L^{+}=L^{-}$.

COROLLARY 3.3.3. The following conditions are pairwise equivalent:

(a) $(X, \pi)$ is a nonwandering flow of characteristics $0^{+}$and 0 .

(b) $D=K^{+}$.

(c) $D=L^{+}$.

(d) $J=K^{+}$.

(e) $D=A_{w}^{+}$.

We now turn to bilaterally Poisson stable flows.

THEOREM 3.4. The following conditions are pairwise equivalent:

(a) $(X, \pi)$ is bilaterally Poisson stable.

(b) $K^{+}=K^{-}$.

(c) $K^{+}=L^{-}$.

(d) $K^{-}=L^{+}$. 
ProOF. The first two conditions were shown to be equivalent in [8]. In that case $C \subset L^{+}=L^{-}$implying the last two conditions. On the other hand, the invariance of the $L^{-}$and $L^{+}$relations yields $C \subset L^{+}=L^{-}$for each of the last two conditions and condition (a) follows. The proof is complete.

There are bilaterally Poisson stable subflows of the toral flow of Example 1 which do not have minimal trajectory closures. Our next theorem characterizes such flows with minimal orbit closures.

THEOREM 3.5. The following conditions are pairwise equivalent:

(a) $(X, \pi)$ is bilaterally Poisson stable and each orbit closure is minimal.

(b) $K^{+}=A_{w}^{-}$.

(c) $K^{+}=A_{w}$.

(d) $K=A_{w}$.

(e) The dual of each condition above.

Proof. First, let condition (a) hold. Then, by Theorem 3.4 and the dual to Theorem 3.2 we have $K^{+}=K^{-}=A_{w}^{-}$. Hence, condition (b) follows.

Next, let condition (b) hold. Since $C^{+} \subset K^{+}=A_{w}^{-}$, we have $C \subset L^{-}$and the flow is negatively Poisson stable. Thus, $L^{-} \subset K^{+}$and $K^{+} \subset L^{-}$implying that $L^{-}=K^{+}=A_{w}^{-}$. The invariance of $L^{-}$implies that $L^{-}=L^{+}$and we obtain bilateral Poisson stability. Hence, $K^{+}=K^{-}=A_{w}^{-}=A_{w}^{+}$by Theorem 3.1 of [8]. Condition (c) follows.

Now, let condition (c) hold. Then, $C^{+} \subset A_{w}$ implies $C \subset L^{+}$. Consequently, the flow is positively Poisson stable and $K=K^{+}=A_{w}$.

Let condition (d) hold. Then, $C \subset A_{w}$, and hence, the flow is bilaterally Poisson stable. Let $y$ be in $K(x)$ for some point $x$ of $X$. By Theorem 3.2 we have $K^{+}(x)$ positively minimal and $K(y)=K^{+}(y)=K^{+}(x)=K(x)$. We conclude that $K(x)$ is minimal (see $[4,4.15, \mathrm{I}]$ ) and (a) holds.

Finally, dual arguments yield the conditions in (e) and the proof is complete.

The final theorem on bilaterally Poisson stable flows is a characterization of such flows of characteristic $0^{ \pm}$. Whenever $X$ is locally compact the flows addressed in the following theorem are the bilaterally Poisson stable flows without saddle limit sets (see [7, 2]).

THEOREM 3.6. The following conditions are pairwise equivalent:

(a) $(X, \pi)$ is a nonwandering flow of characteristic $0^{ \pm}$.

(b) $D^{+}=A_{w}$.

(c) $D^{-}=A_{w}$.

(d) $D=A_{w}$.

ProOF. Each of the conditions implies that $C \subset L$, and hence, that the flow is nonwandering. The Ahmad-Sarabia Theorem 1.1 implies that $D^{+}=D^{-}=D$ making the last three conditions equivalent. It is sufficient to show that conditions (a) and (b) are equivalent in order to complete the proof.

Let condition (b) hold. If $y \in D^{+}(x)=A_{w}(x)$ for some point $x$ of $X$, then $x \in$ $L(y) \subset D^{+}(y)=A_{w}(y)$, and hence, $y \in L^{+}(x) \cap L^{-}(x)$. Thus, we have $D^{+}(x) \subset$ $L^{+}(x)$ and $D^{-}(x) \subset L^{-}(x)$ from which we obtain $D^{+}=K^{+}$and $D^{-}=K^{-}$. Conversely, let $y \in D^{+}(x)$ for some point $x$ of $X$. Then, $x \in D^{-}(y)=J^{-}(y)=$ $L^{-}(y)=L^{+}(y)$ so that $y \in A_{w}(x)$. We have $D^{+}(x) \subset A_{w}(x)$. On the other hand, 
let $y \in A_{w}(x)$. Then, $x \in L^{+}(y)=L^{-}(y) \subset D^{-}(y)$ so that $y \in D^{+}(x)$ yielding $A_{w}(x) \subset D^{+}(x)$. Consequently, $D^{+}=A_{w}$ completing the proof of the theorem.

The next two theorems examine unilaterally Poisson stable flows.

THEOREM 3.7. A flow is unilaterally Poisson stable if and only if $K=L$.

ProOF. The result is evident since $C \subset K=L$ whenever $K=L$ and since $C \subset L^{+} \cup L^{-}$implies $K=L$ whenever the flow is positively or negatively Poisson stable.

THEOREM 3.8. The following conditions are pairwise equivalent.

(a) $(X, \pi)$ is a unilaterally Poisson stable flow of characteristic 0.

(b) $D=L$.

(c) $D^{+}=L$.

(d) $D^{-}=L$.

Proof. Each condition implies that $C \subset L$, and hence, that the flow is nonwandering. Again the Ahmad-Sarabia Theorem 1.1 yields the equivalence of the last three conditions so that we need only show that the first two conditions are equivalent.

If condition (a) holds, then $K=L$ and $D=K$ from which condition (b) follows. Conversely, $C \subset D=L$ implies the flow is Poisson stable in at least one sense. Also $C \subset L$ implies $K=L$, and hence, $D=K$. The proof is complete.

We conclude this section with an example and a characterization of the Poisson stability and recurrent concepts on locally compact spaces using the results of Theorems 1.2 and 1.3.

THEOREM 3.9. Let $X$ be locally compact. The following conditions are pairwise equivalent:

(a) $(X, \pi)$ is positively (negatively, bilaterally) Poisson stable.

(b) $(X, \pi)$ is recurrent.

(c) Any condition of Theorems 3.1, 3.2, 3.4, or 3.5.

COROLLARY 3.9.1. Let $X$ be locally compact. Then the following conditions are pairwise equivalent for a closed invariant set $M$ :

(a) $M$ is a compact minimal set.

(b) $M$ is a positively (negatively) minimal compact set.

(c) $M$ is a positively (negatively, bilaterally) Poisson stable set and $M$ is the closure of some orbit.

(d) $M$ is a recurrent set and $M$ is the closure of some orbit.

COROLlARY 3.9.2. Let $X$ be locally compact. Each nonwandering point is recurrent if and only if each central point is positively (negatively, bilaterally) Poisson stable.

Every periodic or critical point is bilaterally Poisson stable. The following corollary gives a necessary and sufficient condition for a Poisson stable point to be periodic or critical.

CoRollary 3.9.3. A positively (negatively, bilaterally) Poisson stable point is either periodic or critical if and only if its trajectory is a locally compact set. 
EXAMPLE 2. Consider again the toral flow of Example 1. Absolutely no point of $X=T \backslash\{p\}$ is recurrent even though condition (a) of Theorem 3.9 is almost satisfied, i:e. only $C(x)$ and $C(y)$ violate one of the properties of (a). Whenever $C(x)$ and $C(y)$ are removed from $X$, the resulting subflow satisfies conditions (a) and (c) but (b) is totally false. Note also that the subflow on $X \backslash C(x) \cup C(y)$ satisfies condition (c) of Corollary 3.9.1 but all other conditions are false. The toral flow contains subflows showing that the results of Theorem 3.9 and each of its corollaries are sharp.

ACKNOWLEDGEMENT. The author is grateful to the referee for having read this paper with the greatest of care and for suggesting improvements. This research was partially supported by an NMSU Faculty Research Grant.

ADDED IN PROOF. The Poisson stability condition of Theorem 1.2 is insufficient, and hence, 3.9(a), 3.9.1(c), and 3.9.2 do not hold. The author has shown in his unpublished note "Correction of a recurrence and Poisson stability theorem" that these results hold if, in addition to Poisson stability, each point $x$ is approximated by compact weak attractors relative to $K(x)$.

\section{REFERENCES}

1. S. Ahmad, Strong attraction and classification of certain continuous flows, Math. Systems Theory 5 (1971), 157-163.

2. - Dynamical systems of characteristic $0^{+}$, Pacific J. Math. 32 (1970), 561-574.

3. S. Ahmad and J. Sarabia, On nonwandering continuous flows, Funkcial. Ekvac. 21 (1978), 193-201.

4. N. Bhatia and O. Hajek, Theory of dynamical systems, Parts I and II, Tech. Notes BN-599 and BN-606, Univ. of Maryland, 1969.

5. R. Knight, Dynamical systems of characteristic 0, Pacific J. Math. 41 (1972), 368-377.

6. _ Structure and characterizations of certain continuous flows, Funkcial. Ekvac. 17 (1974), 223-230.

7. __ Recurrent and Poisson stable flows, Proc. Amer. Math. Soc. 83 (1981), 49-53.

8. , Dynamically symmetric flows, J. Mathematical and Physical Sci. (1981) (to appear).

9. __ Compact dynamical systems, Proc. Amer. Math. Soc. 72 (1978), 501-504.

10. __ Central motions, Ann. Polon. Math. (to appear).

11. V. Nemytskii and V. Stepanov, Qualitative theory of differential equations, English transl., Princeton Univ. Press, Princeton, N.J., 1960.

Department of Mathematics, Northeast Missouri State University, KirksVILLE, MISSOURI 63501 